\title{
Quasi-static axial crushing of extruded polystyrene foam-filled thin-walled aluminum tubes: Experimental and numerical analysis
}

\author{
L. Aktay ${ }^{\mathrm{a}}$, A.K. Toksoy ${ }^{\mathrm{b}}$, M. Güden ${ }^{\mathrm{b}, \mathrm{c}, *}$ \\ ${ }^{a}$ Institute of Structures and Design, German Aerospace Center (DLR), Pfaffenwaldring 38-40, Stuttgart, Germany \\ ${ }^{\mathrm{b}}$ Department of Mechanical Engineering, Izmir Institute of Technology, Gülbahçe Köyü, Urla, Izmir, Turkey \\ ${ }^{c}$ Center for Materials Research, Izmir Institute of Technology, Gülbahçe Köyü, Urla, 3540 Izmir, Turkey
}

Received 25 June 2004; accepted 6 December 2004

Available online 2 March 2005

\begin{abstract}
The experimental and numerical quasi-static crushing responses of extruded closed cell polystyrene foam-filled thin-walled aluminum tubes were investigated. The numerical crash analysis of empty and foam-filled tubes was performed using the explicit finite element code PAM-CRASH ${ }^{\mathrm{TM}}$. Satisfactory agreements were generally achieved between the finite element model and experimental deformed shapes, load-displacements, fold lengths and specific energy absorptions. The model and experiments have also highlighted the several effects of foam filling on the crushing of thin-walled tubes. The energy absorptions in foam-filled tubes were further shown to be higher than the sum of the energy absorptions of empty tube (alone) and filler (alone).
\end{abstract}

(C) 2005 Elsevier Ltd. All rights reserved.

Keywords: Foams (B); Foam-filled tubes; Finite element modeling

\section{Introduction}

It was first shown in 1960 by Alexander [1] that tubular structures absorb the deformation energy nearly at a constant load. This leads to very high energy absorption efficiencies making these structures suitable for the applications of impact load mitigation. Since Alexander, many experimental and numerical investigations have been conducted aiming at determining and understanding the crushing behavior of tubular structures and some of these have been recently reviewed by Alghamdi [2]. The filling of tubular structures with lightweight foams for a goal of increasing specific energy absorption (SEA) has also taken considerable interest. One of the earliest investigations on the crushing behavior of thinwalled sections filled with lightweight polyurethane

\footnotetext{
* Corresponding author. Tel.: +90 232 7506595; fax: +90 232 7506505.

E-mail address: mustafaguden@iyte.edu.tr (M. Güden).
}

foam was conducted by Thornton [3], who pointed out that although noticeable increase in SEA was possible with foam filling, it was not weight effective when compared with the thickening of empty tube wall. Lampinen and Jeryan [4] investigated the crushing of sheet metal tubes filled with low density polyurethane foams and concluded that foam filling stabilizes the deformation of thin-walled tubes. The crushing behavior of polyurethane foam-filled thin-walled metal tubes was also investigated by Reid et al. [5], at quasi-static and dynamic deformation rates. It was concluded that tube wall interacts with the foam filler deformation resulting in a more tendency for the axisymmetric mode of deformation. They also concluded that the simple addition of the uniaxial foam contribution gave the total average crushing load of filled tube. Guillow et al. [6] have recently pointed out that the average crushing loads of polyurethane foam-filled aluminum (Al) thin-walled tubes were greater than the sum of the average crushing loads of empty tube (alone) and foam (alone), a result which 
contrasted with those of Reid et al. [5] and Reddy and Wall [7]. Seitzberger et al. [8] investigated the crushing behavior of $\mathrm{Al}$ closed-cell foam-filled monotubal and bitubal arrangements of square, hexagonal and orthogonal cross-sections and concluded that mass related average load level can be improved by filling tubular members with aluminum foam. It was also pointed out in the same study that suitable foam vs. tube selection was important for the designing of efficient crush elements. Santosa and Wierzbicki [9] investigated the crushing behavior of $\mathrm{Al}$ honeycomb and foam-filled box columns, both numerically and experimentally, and showed that the effect of filler on the tube crushing load was similar when the strong axis of the honeycomb through and normal to the compression axis, proving that both axial and lateral strength of the filler were effective in increasing the crushing load of the tube. Further, Santosa et al. [10] noted that the bonding between filler and tube wall increased the average crushing load of filled tube over the unbounded filled tube when appropriate tube geometry and foam density were chosen. They also showed, both experimentally and numerically, that the strengthening of foam-filled tube average crushing load was 1.8 and 2.8 times of Al foam filler plateau load for the unbounded and bounded cases, respectively.

In this study, we report experimental and numerical analysis of quasi-static crushing of extruded polystyrene foam-filled Al thin-walled tubes. Polystyrene foams are currently used as the filler in the inner layer of motorcyclist's helmets for its impact-protection function, but their capabilities in thin-walled tubes as lightweight filler materials have not been investigated and modeled yet. As compared with expanded counterparts, extruded polystyrene foams have more homogenous cell size and contain no weak boundaries of beads; hence potentially more suitable materials for the filling of thin tubular sections. Selected two thin-walled Al tubes having different diameters and wall thicknesses and deforming in diamond mode of deformation were filled with two different densities of polystyrene foam in order to determine the effect of foam filling and foam filler density on the crushing behavior of tubes. Although the present report focussed on the simulation of the experimental crushing behavior of tubes, the results were also compared with those previous investigations on the foamfilled thin-walled tubes.

\section{Materials and compression testing}

The selected Al thin-walled tubes $(99.7 \% \mathrm{Al})$, produced by Metalum Company of Turkey using a cold deep drawing process, were $16 \mathrm{~mm}$ in outer diameter with a wall thickness of 0.23 and $25 \mathrm{~mm}$ in outer diameter with a wall thickness of $0.29 \mathrm{~mm}$. Typical quasi-sta-
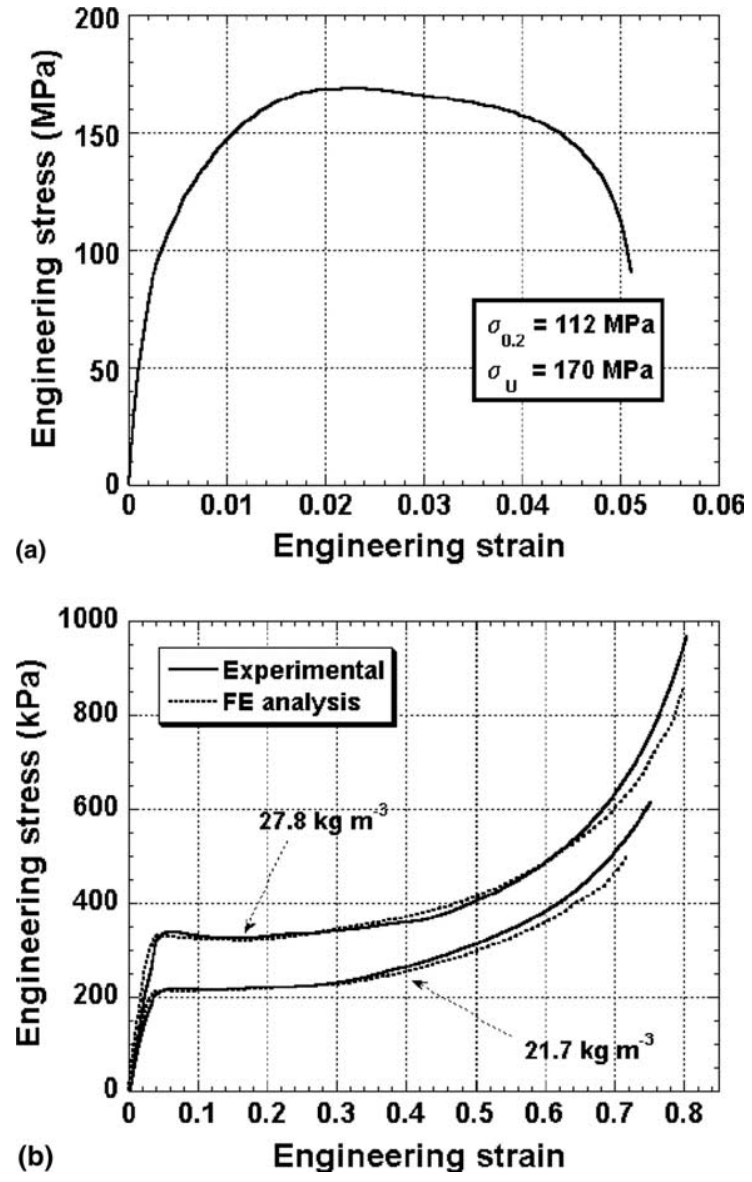

Fig. 1. (a) Tensional engineering stress-strain curve of Al tube material and (b) experimental and FE quasi-static compression stress-strain curves of polystyrene foam fillers.

tic $\left(1 \times 10^{-3} \mathrm{~s}^{-1}\right)$ engineering tensile stress-strain curve of tube material tested in accordance with ASTM B557M standard is shown in Fig. 1(a). Relatively, high strain hardening seen in the inelastic deformation region of the curve is most likely due to cold forming process applied. Tubes were filled and tested without any heat treatment applied.

Commercially available extruded polystyrene foam sheets, manufactured by Izocam Company of Turkey, were in two different average densities: 21.7 and $27.8 \mathrm{~kg} \mathrm{~m}^{-3}$. Uniaxial compression stress-strain curves of foams determined in accordance with ASTM 162191 on $40 \mathrm{~mm}$ long cubic samples are shown in Fig. 1(b). The curves show typical elastoplastic foam compression behavior, composing of three distinct deformation regions: elastic, collapse (plateau) and densification. Detailed information on the compression testing and assessment of mechanical properties of the foams are given elsewhere [11].

Cylindrical foam fillers with an outer diameter that was slightly larger than the inner diameter of empty tube $(\sim 0.5 \mathrm{~mm})$ were core drilled from the as-received polystyrene foam sheets through the thickness direction. 


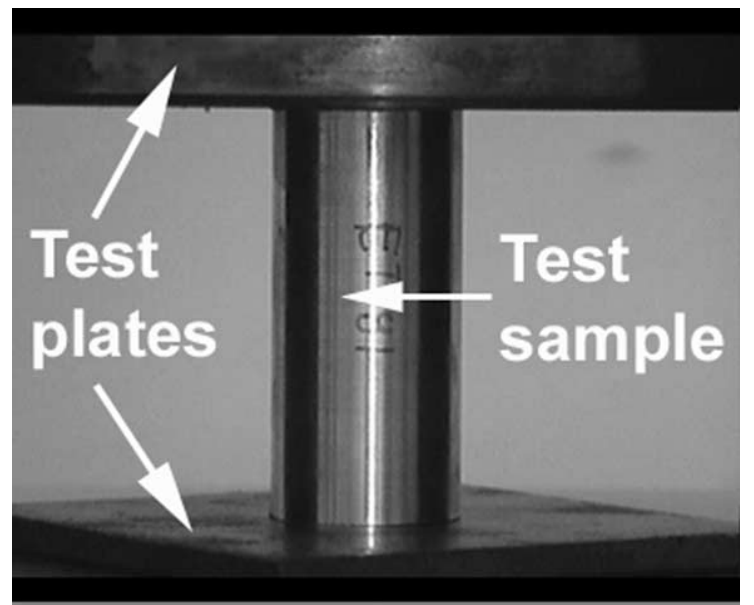

Fig. 2. Uniaxial compression test set-up, test sample (16-mm empty tube) and compression test plates.

The foam filler samples were inserted into empty tubes tightly without imposing any damage to the cells. Compression tests were conducted on a fully computer controlled Schmadzu AGI universal testing machine. Test samples were compressed between two flat compression test plates without any constraint applied to the ends of tubes as shown in Fig. 2. Compression tests on empty and foam-filled tubes, all $40 \mathrm{~mm}$ in length, were performed at a deformation rate of $100 \mathrm{~mm} \mathrm{~min}^{-1}$, same with that of compression tested foam samples.

\section{Modeling studies}

The geometrical models used in the modeling were created in commercial finite element package ANSYS ${ }^{\mathrm{TM}}$. Later the meshed geometrical models were exported to PAM-GENERIS ${ }^{\mathrm{TM}}$ in order to set the boundary conditions. The numerical solutions were carried out using the explicit finite element code PAM-CRASH ${ }^{\mathrm{TM}}$. PAMVIEW $^{\text {TM }}$ was used as the visualization tool postprocessor.

The aluminum tubes were modeled using BelytschkoTsay-4 node-thin shell elements. In foam-filled tube modeling, only one half of the tube was modeled due to symmetry. The foam filler and the compression test plates were modeled with 8-node solid elements. Finite element meshes of the quasi-static axial compression testing of empty and foam-filled tubes are shown in Fig. 3(a) and (b), respectively.

The compression test base and upper plates were modeled as rigid body and kinematical boundary. The
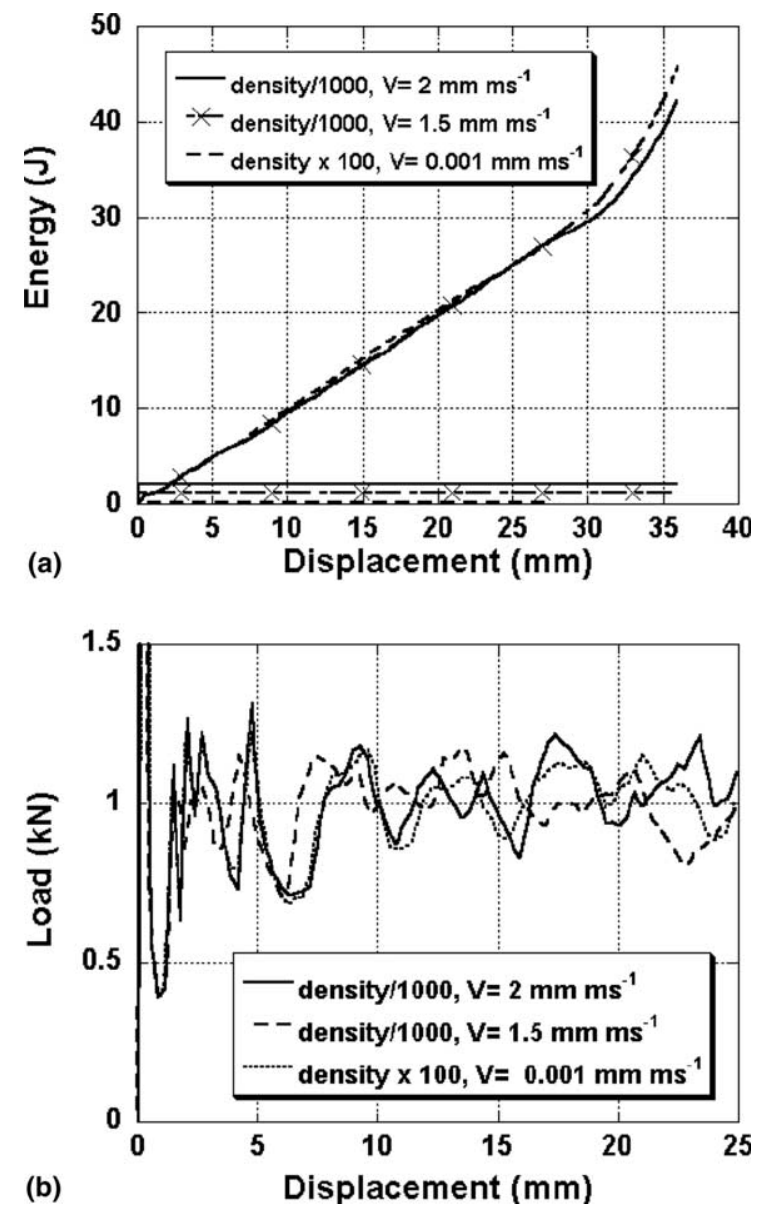

Fig. 4. (a) Kinetic and internal energy histories of 16-mm empty tube at various density scaling and deformation rates and (b) corresponding load-displacement curves.

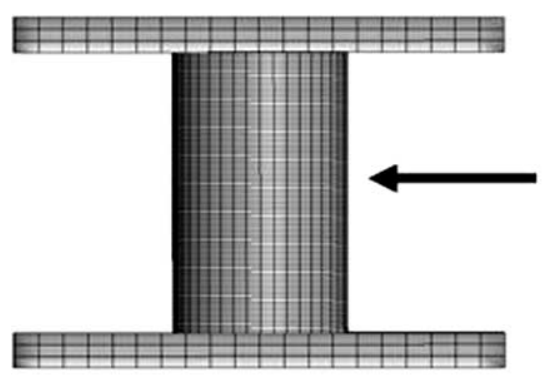

(a)
(1)

(3)

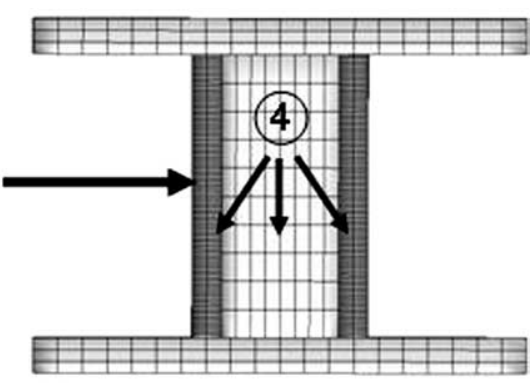

(b)

Fig. 3. FE meshing of: (a) empty tube (full model) and (b) foam-filled tube (half model), 1 and 2:compression test plates, 3: empty and foam-filled tube meshing and 4 : foam filler meshing. 
displacements and rotations of the compression test plates were not allowed except the displacement of the upper plate along the vertical axis. The movement of the rigid body was fully determined by the movement of an artificial "node" located at the rigid body center of gravity. Boundary conditions for the rigid body were applied to the center of gravity node only.

Material type 103 corresponding to the elastic-plastic isotropic thin shell material model was used for the tube material. Material type 103 uses an enhanced plasticity algorithm that includes transverse shear effects. It exactly satisfies Hill's criterion and precisely updates the element thickness during the plastic deformation. The base and upper plates were modeled using an elasticplastic solid element model called material type 1 . This material model corresponds to elastic-plastic behavior with isotropic hardening and elastic-plastic behavior can be introduced by specifying the yield stress and tangent modulus or specifying the effective plastic strains and stresses.

The material behavior of polystyrene foam was modeled using the crushable foam solid model, namely material type 2. Material type 2 corresponds to the solid materials exhibiting coupled volumetric (bulk) and deviatoric (shear) plasticity. The coupling between both parts of the material response is established by a pres- sure dependent von Mises' yield surface. Prior to the numerical investigation of foam-filled tubes, polystyrene foam compression behavior was simulated. The numerical simulations and experimental results were compared for each density of polystyrene foam and the comparison is shown in Fig. 1(b). Agreement between numerical and experimental stress-strain curves shown in this figure is satisfactory for the modeling purpose.

Three contact models were used in the modeling: (i) a tied contact between the base plate and the empty tube, (ii) a node-to-segment contact between the upper plate and the tube ends and (iii) a self-contact (self impacting contact with edge treatment) in order to prevent the interpenetration between folds in the tube wall during plastic deformation. The self-contact impact algorithm of type 36 allows all slave segments defined in a given sliding interface. No segment orientation is needed to be specified, since the algorithm automatically detects penetrations and keeps in memory the segment side from which a slave node comes into contact. The selfcontact impact algorithm also uses a search algorithm so-called $3 D$ Bucket sort algorithm in which the 3D slave surface is subdivided into a number of buckets and the slave nodes are recalculated in terms of bucket coordinates. According to a slave node bucket coordinates, its proximity to slave segments is quickly determined.
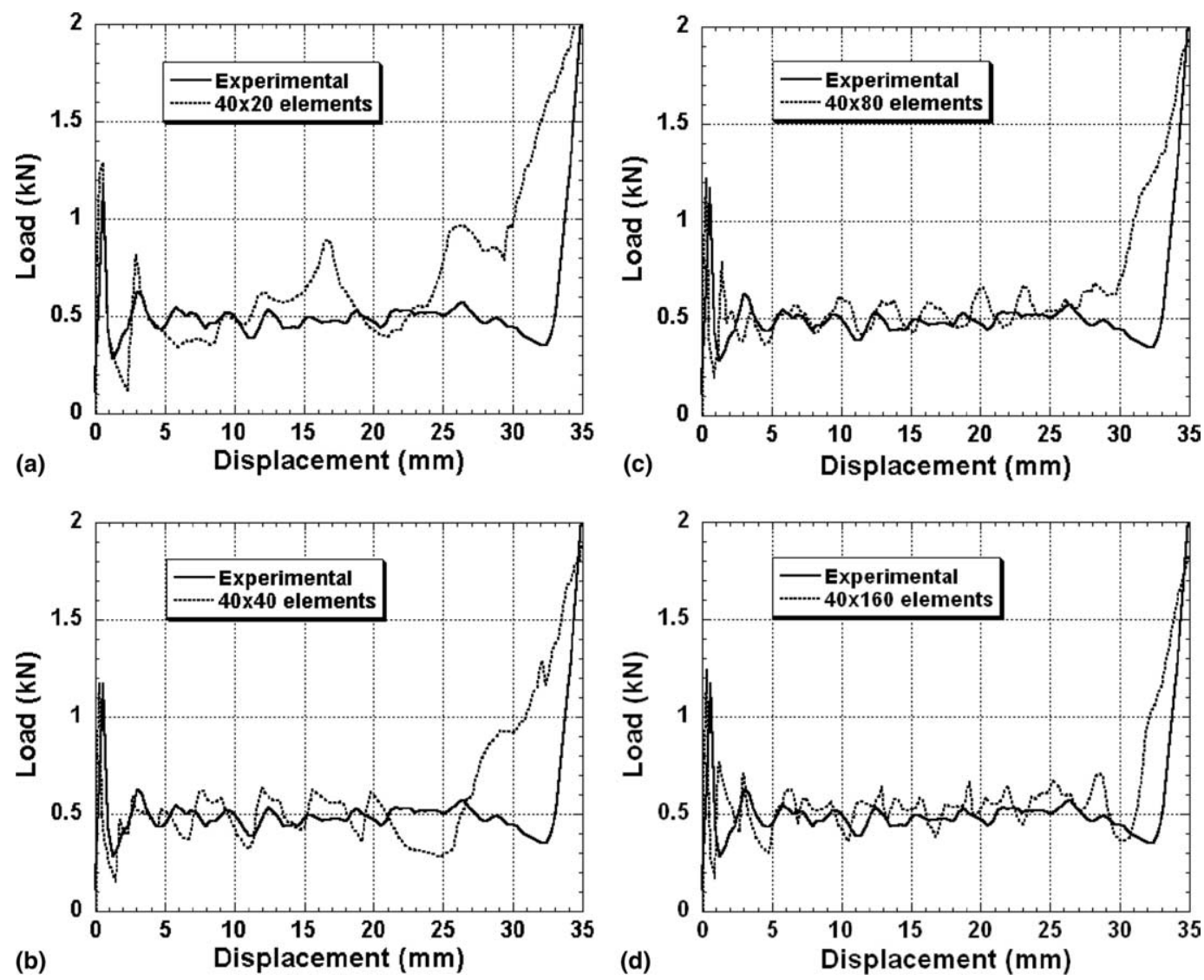

Fig. 5. Numerical and experimental load-displacement curves of $16-\mathrm{mm}$ empty tube for different meshes along the height: (a) $40 \times 20$, (b) $40 \times 40$, (c) $40 \times 80$ and (d) $40 \times 160$. 
After the global search phase, an accurate local search algorithm is started.

The folding of the tube wall results in local deformation of and penetration to the foam filler surface. It was
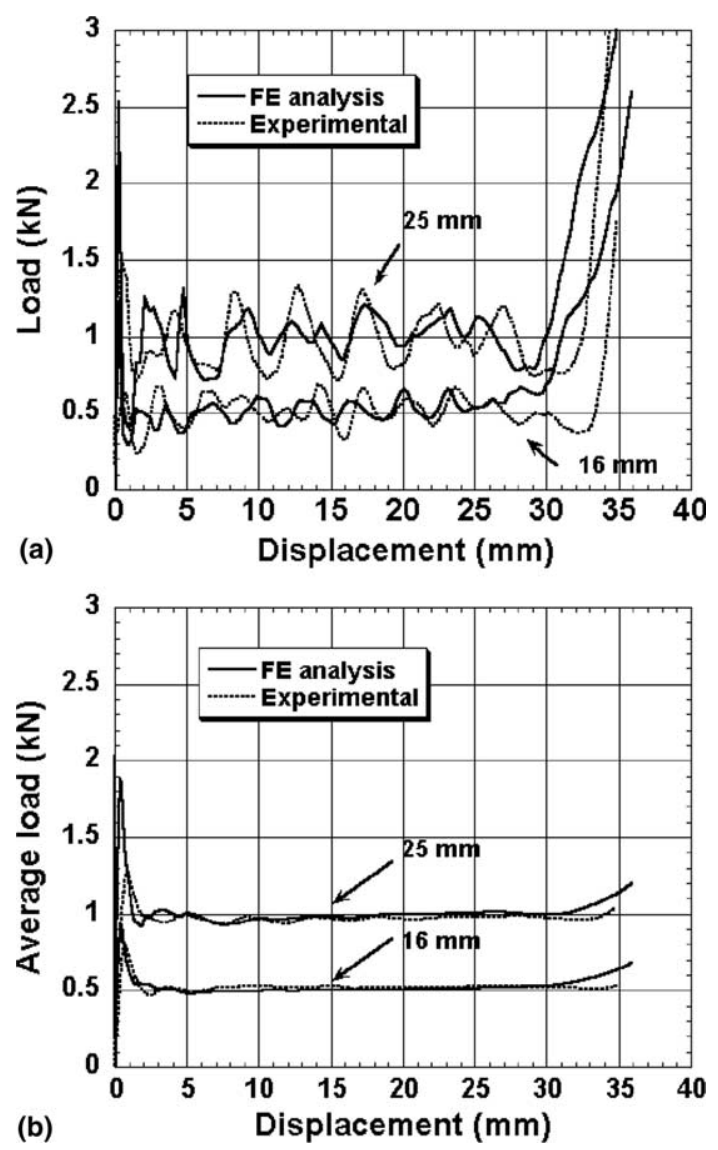

Fig. 6. Comparison of experimental and numerical: (a) load-displacement and (b) average load-displacement curves of 16- and 25-mm empty tubes. experimentally observed that the penetration depth through the foam filler surface was relatively small as compared with the radius of the filler. Based on this observation, a fine discretization was applied, in order to reduce the computational time, to the foam filler near the tube wall surface as shown in Fig. 3(b). The mesh patterns combined with a tied kinematic constraint type contact that connects two contact interfaces defined on two meshed parts of a structure that are close to each other but whose respective finite element grids are not necessarily matching. Internal solid anti-collapse contact, namely contact type 10 , was used additionally in order to prevent numerical problems that could arise when the solid elements of foam were heavily compressed and distorted. The option helps to reduce excessive compression; therefore, avoids too large drops in the stable solution time steps. This option also helps to avoid too large element distortions that may lead to ill-defined elements causing negative volume. The interaction between the tube wall and the foam filler was defined via multiple segment-to-segment contact, type 23 . Multiple segment-to-segment contact uses an enhanced searching algorithm for detecting penetrations. Based on the each master segment domain box, candidate slave nodes are searched by an efficient sorting method. For a given master segment at every time step, only its candidate nodes are checked for penetrations. Penalty forces are applied to those nodes which are judged to be penetrating the master segment.

\subsection{Mesh optimization}

In finite element analysis, the number of finite element mesh for a fixed geometry leads to variation in element size and numerical results. The optimal finite

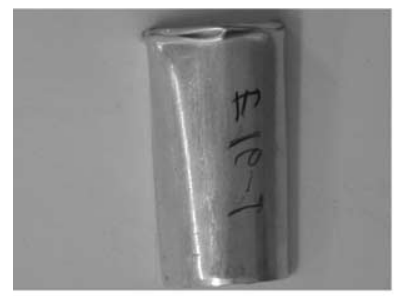

(a)

$15 \%$

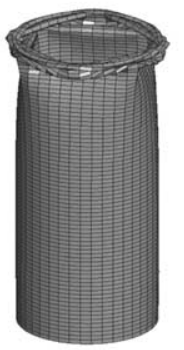

$15 \%$

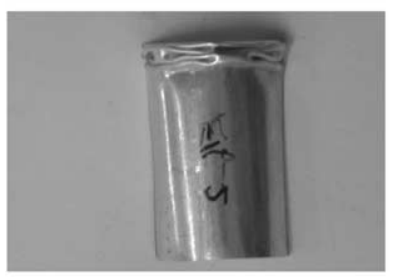

$30 \%$

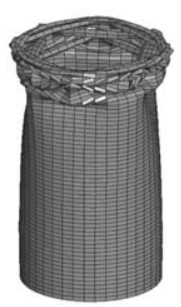

$30 \%$

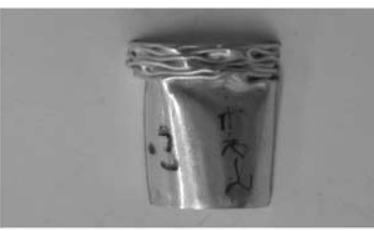

$50 \%$

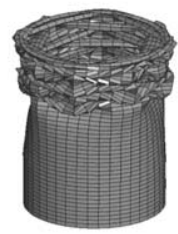

$50 \%$

Fig. 7. Deformation histories of 16-mm empty tube at various percent deformation ratios: (a) experimental and (b) numerical. 
element mesh number has been established by refining the mesh till convergence is reached. For both geometries, 16 and $25 \mathrm{~mm}$, the circumference was meshed with 40 elements and in order to determine the optimal number of mesh, both tubes were meshed with $20,40,80$ and 160 elements along the height. The nomenclature used to describe the models is that $40 \times 80$ is to be interpreted as 40 elements along the circumference and 80 elements along the height.

\subsection{Quasi-static simulation}

The compression of empty and foam-filled tubes was performed at a cross-head speed of $100 \mathrm{~mm} \mathrm{~min}^{-1}$, corresponding to a deformation rate of $4.16 \times 10^{-2} \mathrm{~s}^{-1}$ (the cross-head speed divided by the initial length of the tube) which is relatively slow for the dynamic numerical simulations, since the explicit time integration method is conditionally stable when time step is sufficiently small. Therefore, in general, very small time increments have to be used. Contrary to implicit method, which is more efficient for relatively slow phenomena such as vibration types of problems, the explicit method is more efficient for very fast phenomena such as impact and explosion. Santosa et al. [10], however, presented a procedure to achieve a quasi-static process using the explicit dynamics scheme by suggesting two alternatives; scaling down the material mass and scaling up the mass density. The first one, scaling down the mass, results in large number of time increments. The second alternative, scaling up the mass density, results in larger time step and therefore reduces the number of time step increment for such a low loading rate. In this present work, the loading velocity was scaled up to $2 \mathrm{~mm} \mathrm{~ms}^{-1}$ and the material density was scaled down by a factor of 1000 . The loading velocity was reached first $0.01 \mathrm{~ms}$ of the simulation and then was hold constant.

As stated in the work Santosa et al. [10], above explained procedure requires the control of two simulation responses in order to verify the quasi-static process is hold. First, the total kinetic energy should be very small as compared with the total internal energy over the period of crushing response. Second, the crushing load-displacement response of the system must be an independent function of the applied velocity. Therefore, kinetic and internal energy histories were carefully investigated using different mass density scaling and deformation velocities. Fig. 4(a) shows that kinetic energy is relatively smaller than the internal energy for the investigated three different mass density scaling-deformation rates combinations. It is also shown in Fig. 4(b) that the load-displacement responses of density-rate combinations are very similar. Based on efficiency and solution time considerations, numerical implementations were decided to be performed by scaling down the mass density with a factor of 1000 and with a loading velocity of $2 \mathrm{~mm} \mathrm{~ms}^{-1}$.

\section{Results and discussions}

\subsection{Empty aluminum tubes}

The load-displacement curves of empty tube models constructed with four different numbers of elements; $40 \times 20, \quad 40 \times 40, \quad 40 \times 80$ and $40 \times 160$, are shown sequentially in Figs. 5(a)-(d) for 16-mm tube, respectively. In these figures, for the purpose of comparison

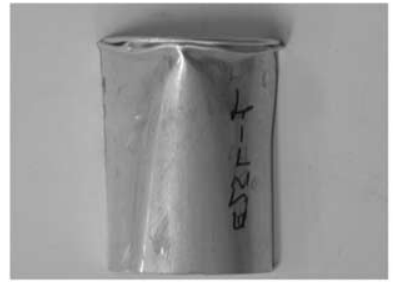

(a)

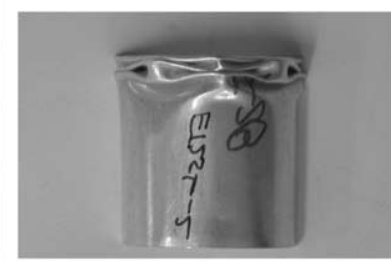

$30 \%$

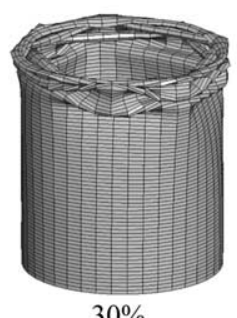

$30 \%$

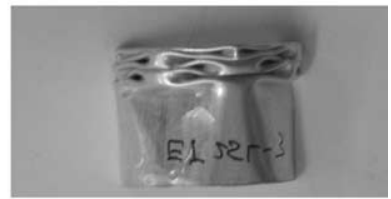

$50 \%$

(b)

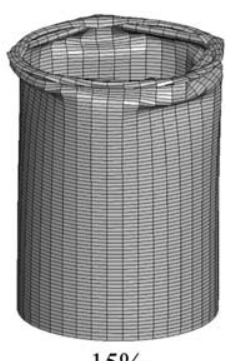

$15 \%$

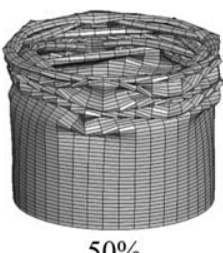

Fig. 8. Deformation histories of 25-mm empty tube at various percent deformation ratios: (a) experimental and (b) numerical. 
experimental load-displacement curve of 16-mm empty tube is also shown. As seen in Figs. 5(c) and (d), the load-displacement curves of models with $40 \times 80$ and $40 \times 160$ elements give reasonably good agreements with the experimental load-displacement curve, while the load-displacement curves of models constructed with $40 \times 20$ and $40 \times 40$ elements show substantial disagreements. The similar effects of number of elements on the load-displacement curves of $25-\mathrm{mm}$ empty tube were also found. Finite element models were, however, continued with $40 \times 80$ elements for both tube geometries for the computational efficiency.

It was experimentally and numerically found that both 16- and 25-mm empty tubes deformed in diamond mode and corresponding experimental and numerical load-displacement and average load-displacement curves are shown in Figs. 6(a) and (b), respectively. The agreement between experimental and numerical average load values is quite satisfactory except in the initial and densification regions of the load-displacement curves, corresponding to the points of the first and the last fold formation. Totally, 9-10 and 7-8 folds formed in 16- and 25-mm empty tubes, respectively. The experimental and model deformed shapes of empty 16 and $25 \mathrm{~mm}$ tubes corresponding to the deformation ratios of $15 \%, 30 \%$ and $50 \%$ are sequentially shown in Figs.

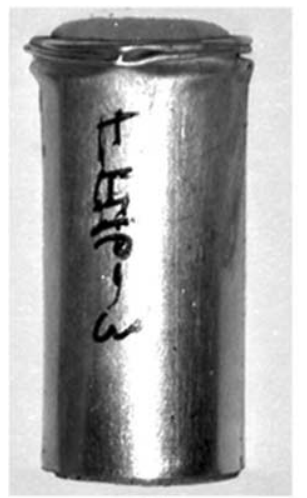

(a) $15 \%$

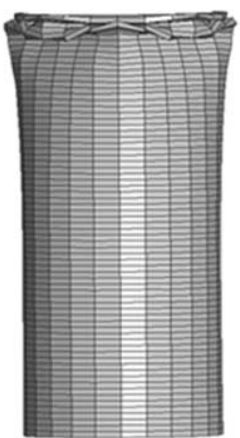

(b)

$15 \%$

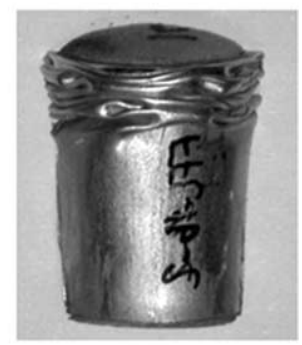

$50 \%$

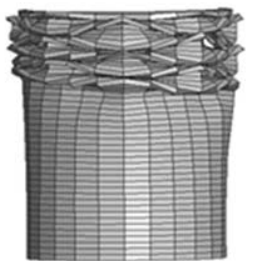

$50 \%$
Fig. 9. Deformation histories of $16-\mathrm{mm}$ foam-filled $\left(27.8 \mathrm{~kg} \mathrm{~m}^{-3}\right)$ tube at $15 \%$ and $50 \%$ deformations: (a) experimental and (b) numerical.
7 and 8. The model deformed shapes exactly matched to the experimental deformed shapes, which again confirmed the agreement between model and experiments. It is also noted in model and experimental deformed shapes of Figs. 7 and 8 that folding started in axisymmetric mode before reverting into diamond mode in both tubes, a phenomenon observed in multi-mode collapse mode [12].

\subsection{Filled aluminum tubes}

In 16-mm foam-filled tube, both model and experiment resulted in diamond mode of collapse same as the collapse mode of empty tube. Figs. 9(a) and (b) show the agreements between the experimental and model deformed shapes of tubes at $15 \%$ and $50 \%$ deformations. The effect of foam filling is to reduce the fold length and hence to increase the number of folds formed (10 11 ), a result which was also previously found in foamfilled Al and steel tubes [8-10,13]. The load values of model further show good coincidence with those of experiment as depicted in Fig. 10. In contrast to 16$\mathrm{mm}$ tube, the foam filling in $25-\mathrm{mm}$ tube switched the collapse mode from diamond to concertina mode and again model and experiment show very similar deformed shapes (Fig. 11) and load-displacement curves (Fig. 12). The reduction in fold length in filled tube also increased the number fold to $9-10$.

The restraining effect of foam filler to the fold formation was explained to be the major cause of the reduced fold length in filled tubes [9]. The entrance of the column wall into foam filler results in shorter fold lengths and increases the number of fold formed [9]. Santosa et al. [10] stated that the encroachment of the column wall into the $\mathrm{Al}$ foam filler allows an additional compression in the foam and retards the sectional collapse of the column. A similar filler-tube encroachment effect is also seen on the surface images of partially crushed foam-

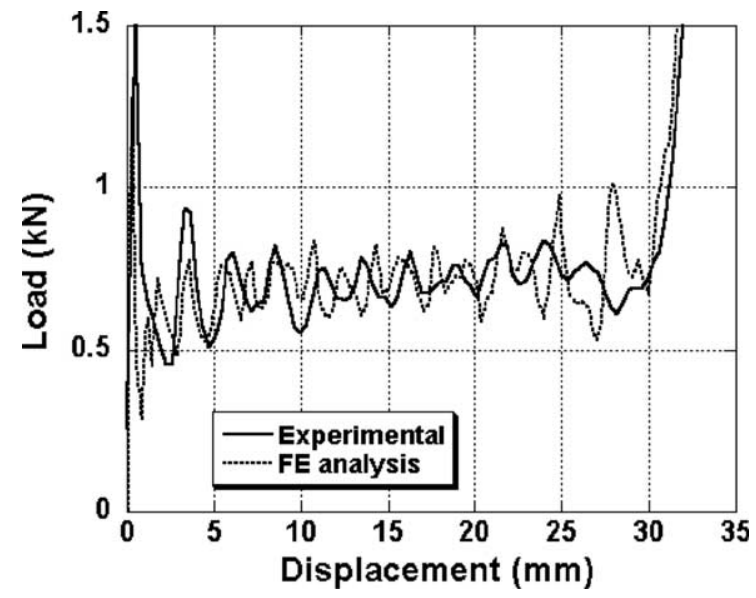

Fig. 10. Numerical and experimental load-displacement curve of $27.8 \mathrm{~kg} \mathrm{~m}^{-3}$ polystyrene foam-filled $16-\mathrm{mm}$ tube. 


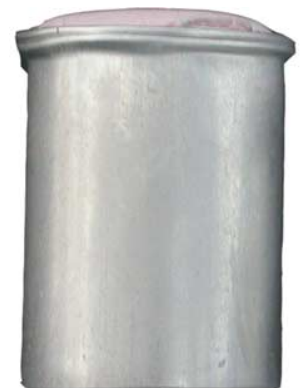

(a) $15 \%$

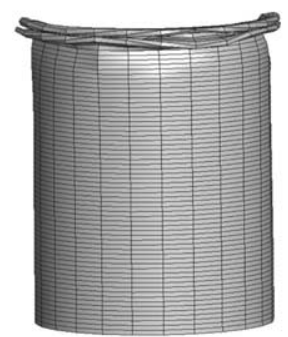

(b)

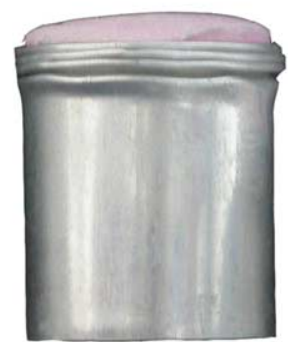

$30 \%$

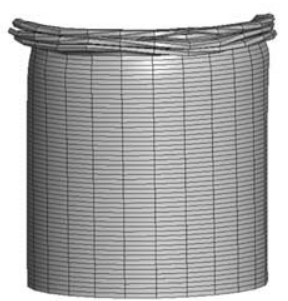

$30 \%$

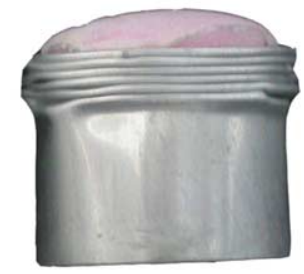

$50 \%$

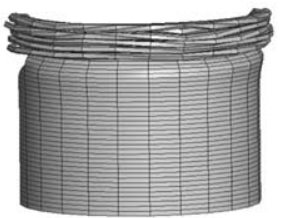

$50 \%$

Fig. 11. Deformation histories of $25-\mathrm{mm}$ foam-filled $\left(27.8 \mathrm{~kg} \mathrm{~m}^{-3}\right)$ tube at various percent deformation ratios: (a) experimental and (b) numerical.

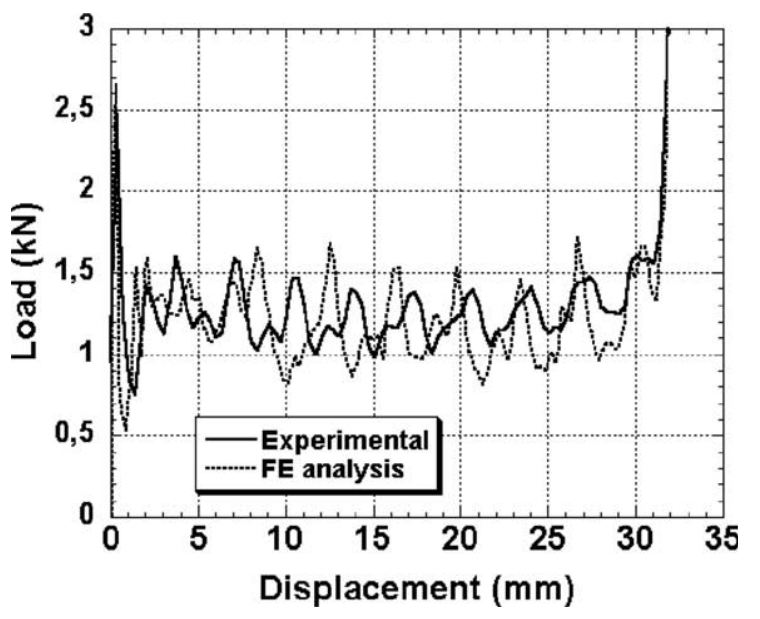

Fig. 12. Numerical and experimental load-displacement curves of $27.8 \mathrm{~kg} \mathrm{~m}^{-3}$ polystyrene foam-filled $25-\mathrm{mm}$ tube.

filled tube's fillers and tubes in Figs. 13(a) and (b). For comparison the experimental and numerical fold lengths of tubes as function of foam density are shown in Fig. 14. The numerically measured fold lengths are slightly higher than the experimentally measured fold lengths and the effect of increasing foam density is to reduce the fold length as seen in Fig. 14, confirming the restraining effect of foam filler to tube wall folding; higher the density of foam filler higher the resistance to tube wall folding. A higher degree of reduction in the fold length of $25-\mathrm{mm}$ tube as compared with that of 16-mm tube may be considered as a signal for the collapse mode-shift, since the number of concertina rings (a)

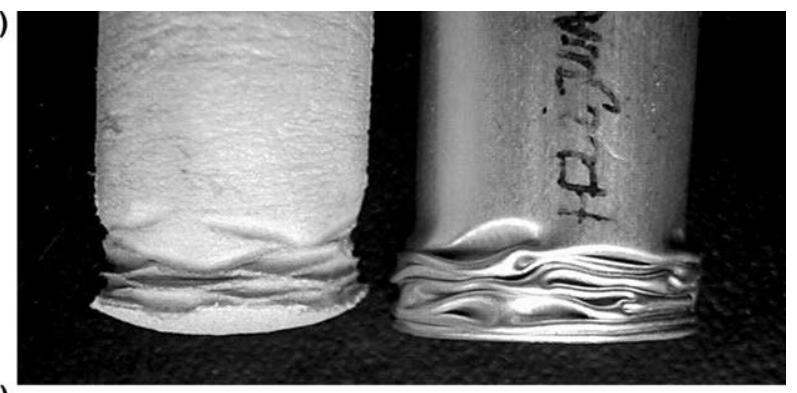

(b)

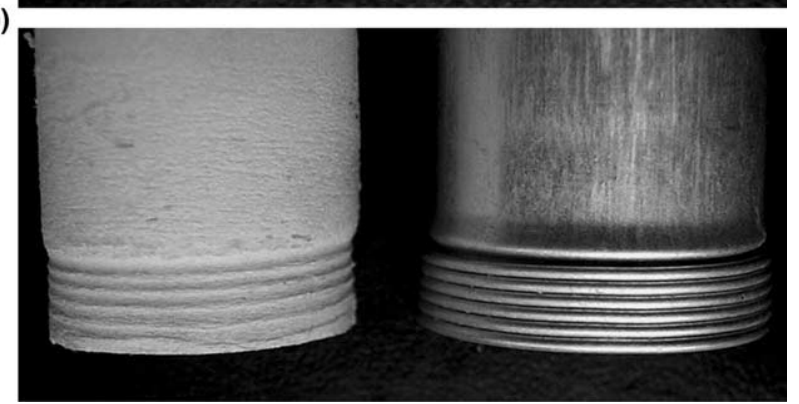

Fig. 13. Images of side views of partially deformed $27.8 \mathrm{~kg} \mathrm{~m}^{-3}$ polystyrene foam-filled tubes at $30 \%$ deformation; filler and tube surfaces: (a) $16 \mathrm{~mm}$ and (b) $25-\mathrm{mm}$ tube.

possibly is higher than the diamond mode for a fixed length[12,14].

The change of collapse mode from diamond into concertina with foam filling was also observed previously in Al foam-filled $\mathrm{Al}$ and steel tubes, polyurethane foamfilled Al tubes and wood sawdust-filled plastic tubes $[6,10,15-17]$ and it was proposed to be due to the thickening effect of foam filling, which drives the deformation 


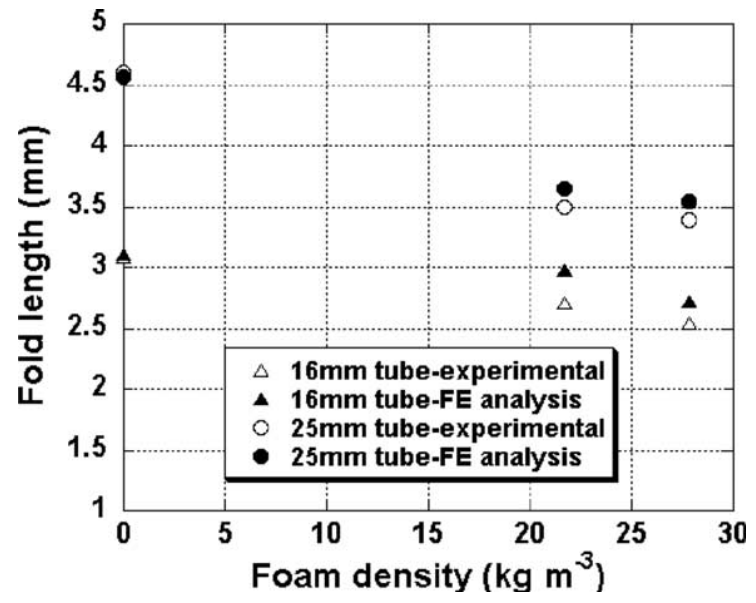

Fig. 14. Numerical and experimental fold lengths vs. foam density.

shift from diamond to concertina. It was also shown by Hanssen et al. [18] that after a critical Al foam density the deformation mode in filled $\mathrm{Al}$ tubes shifted from diamond to concertina mode. A similar mode-shift was also found in polyurethane foam-filled thin-walled Al tubes with the increasing of foam density [6]. For the present work, it is quite possible that the foam densities used are higher than the critical foam density for the modeshift in 25-mm tube and vice verse in 16-mm Al tube.

The experimental and numerical SEAs corresponding to the $50 \%$ deformation of empty and foam-filled tubes are shown squentially for 16- and 25-mm tubes in Figs. 15(a) and (b). Again, experimental and numerical SEAs are well agreed with each other; the maximum differences between them are $6 \%$ and $2 \%$ for $16-$ and $25-\mathrm{mm}$ tubes, respectively. It is also noted in Figs. 15 that lower density foam filling $\left(21.7 \mathrm{~kg} \mathrm{~m}^{-3}\right)$ is almost ineffective in increasing the SEAs of the filled tubes over those of empty ones. The percentage increase in SEA in higher density foam filling $\left(27.8 \mathrm{~kg} \mathrm{~m}^{-3}\right)$ are found numerically $14 \%$ and experimentally $21 \%$ for $16-\mathrm{mm}$ tube and numerically $1.5 \%$ and experimentally $5 \%$ for $25-\mathrm{mm}$ tube. These results also show agreement with the study of Guillow et al. [6] in that the average crushing load of foam-filled tube is higher than that of simply addition of crushing loads of empty tube (alone) and foam filler (alone). It should, however, be stated here that the comparison of the energy absorptions between empty and filled tubes should be made on the equal mass basis by taking into consideration the thickening of the empty tube wall. Previous studies on Al foam-filled tubes and boxes have shown that there existed a critical total filled tube mass and the corresponding critical foam density above which the use of foam filling became more efficient than empty tube $[9,13,19-21]$. The effect of polystyrene foam filler density and the critical total mass of filled tubes should be further investigated numerically and experimentally for efficient crash element designs.
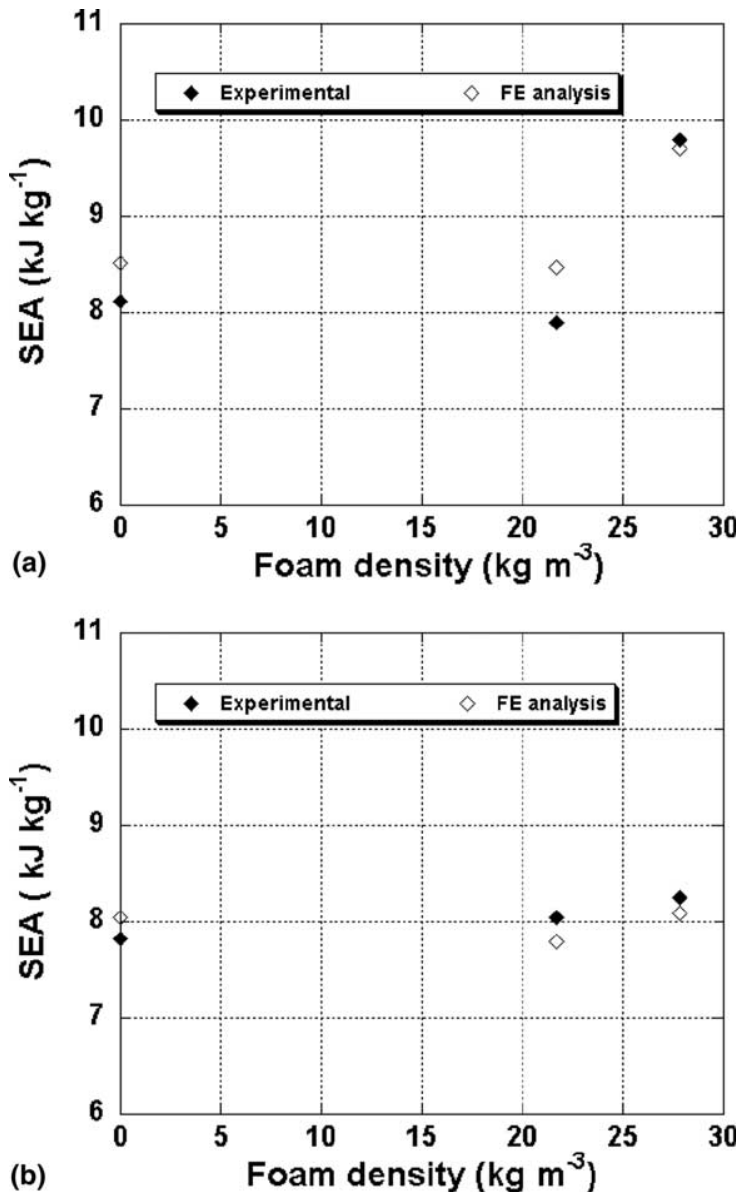

Fig. 15. Numerical and experimental SEAs (at 50\% deformation) in tubes as function of foam density: (a) $16 \mathrm{~mm}$ and (b) $25-\mathrm{mm}$ tube.

\section{Conclusions}

The quasi-static crushing behavior of extruded polystyrene foam-filled $\mathrm{Al}$ tubes was investigated experimentally and numerically. The numerical solutions were carried out using the explicit finite element code PAM$\mathrm{CRASH}^{\mathrm{TM}}$. The kinetic and internal energy histories were investigated at different mass density scaling and deformation velocities and based on efficiency and time inexpensive modeling a mass density scaling with a factor of 1000 and a loading velocity of $2 \mathrm{~mm} \mathrm{~ms}^{-1}$ were chosen for the modeling. In general, satisfactory agreements were found between the results of FE modeling and experimental quasi-static crushing tests of empty and polystyrene foam-filled thin-walled Al tubes. The model and experiments have also highlighted several effects of foam filling in thin-walled $\mathrm{Al}$ tubes. The number of folds formed in foam-filled tubes, both in diamond and concertina mode of deformation, increased with foam filling and also with increasing foam filler density. It was further found that the restraining effect of filler shifted the deformation mode from diamond to concertina in the larger diameter tube investigated. The energy absorptions in foam-filled tubes were shown to increase 
with increasing filler density and higher than the sum of the energy absorptions of empty tube (alone) and filler (alone).

\section{Acknowledgments}

The authors thank the Scientific and Technical Council of Turkey (TUBITAK) for the Grant \#MISAG-227.

\section{References}

[1] Alexander JM. An approximate analysis of collapse of thin-walled cylindrical shells under axial loading. Q J Mech Appl Math 1960;13:1-9.

[2] Alghamdi AAA. Collapsible impact energy absorbers: an overview. Thin Wall Struct 2001;39:189-213.

[3] Thornton PH. Energy absorptions by foam filled structures. SAE paper $800372 ; 1980$.

[4] Lampinen BH. Jeryan RA. Effectiveness of polyurethane foam in energy absorbing structures. SAE paper 820494; 1982.

[5] Reid SR, Reddy TY, Gray MD. Static and dynamic axial crushing of foam filled sheet metal tubes. Int $\mathbf{J}$ Mech Sci 1986;23:295-322.

[6] Guillow SR, Lu G, Grezbieta RH. Quasi-static compression of thin-walled circular aluminum tubes. Int $\mathbf{J}$ Mech Sci 2001;43:2103-23.

[7] Reddy TY, Wall RJ. Axial compression of foam filled thin-walled circular tubes. Int J Impact Eng 1988;7:151-66.

[8] Seitzberger M, Rammerstorfer FG, Gradinger R, Degischer HP, Blaimschein M, Walch C. Experimental studies on the quasi-static axial crushing of steel columns filled with aluminum foam. Int $\mathbf{J}$ Solids Struct 2000;37:4125-47.
[9] Santosa S, Wierzbicki T. Crash behavior of box columns filled with aluminum honeycomb or foam. Comput Stuct 1998;68:343-67.

[10] Santosa S, Wierzbicki T, Hanssen AG, Langseth M. Experimental and numerical studies of foam-filled sections. Int J Impact Eng 2000;24:509-34.

[11] Toksoy AK. Quasi static axial compression behavior of empty and polystyrene foam-filled aluminum tubes. Master thesis, Department of Mechanical Engineering, Izmir Institute of Technology; 2003.

[12] Singace AA, El-Sobky H. Interplay of factors influencing collapse modes in axially crushed tubes. Int J Crash 2000;5:279-97.

[13] Seitzberger M, Rammerstorfer FG, Degischer HP. Crushing of axially compressed steel tubes filled with aluminum foam. Acta Mech 1997;125:93-105.

[14] Andrews KRF, England GL, Ghani E. Classification of the axial collapse of cylindrical tubes under quasi-static loading. Int J Mech Sci 1982;25:687-96.

[15] Hanssen AG, Langseth M, Hopperstad OP. Static and dynamic crushing of square aluminium extrusions with aluminum foam filler. Int J Impact Eng 2000;24:347-83.

[16] Hanssen AG, Hopperstad OS, Langseth M. Design of aluminum foam-filled crash boxes square and circular cross-sections. Int $\mathbf{J}$ Crash 2001;6:177-88.

[17] Singace AA. Collapse behaviour of plastic tubes filled with wood sawdust. Thin Wall Sturct 2000;37:163-87.

[18] Hanssen AG, Langseth M, Hopperstad OP. Static and dynamic crushing of circular aluminum extrusions with aluminum foam filler. Int J Impact Eng 2000;24:475-507.

[19] Toksoy AK, Guden M, Tanoglu M, Hall IW. Effect of adhesive on the strengthening of foam-filled circular tubes. J Mater Sci 2004;39:1503-6.

[20] Hanssen AG, Langseth M, Hopperstad OP. Optimum design for energy absorption of square aluminium columns with aluminum foam filler. Int J Mech Sci 2001;43:153-76.

[21] Seitzberger M, Willminger S. Application of plastic collapse mechanisms for the axial crushing analysis of tubular steel structures filled with aluminum foam. Int J Crash 2001;6:165-76. 